\title{
Evidence-based guideline: Intravenous immunoglobulin in the treatment of neuromuscular disorders
}

Report of the Therapeutics and Technology Assessment Subcommittee of the American Academy of Neurology

0 미슷

H.S. Patwa, MD

V. Chaudhry, MD

H. Katzberg, MD

A.D. Rae-Grant, MD

Y.T. So, MD, PhD

Correspondence \& reprint requests to American Academy of Neurology:

guidelines@aan.com
Supplemental data at www.neurology.org

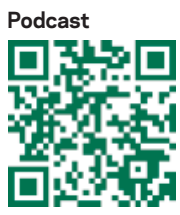

Supplemental Data
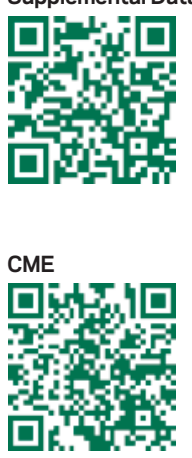

\section{ABSTRACT}

Objective: To assess the evidence for the efficacy of IV immunoglobulin (IVIg) to treat neuromuscular disorders.

Methods: The MEDLINE, Web of Science, and EMBASE databases were searched (1966-2009). Selected articles were rated according to the American Academy of Neurology's therapeutic classification of evidence scheme; recommendations were based on the evidence level.

Results and Recommendations: IVIg is as efficacious as plasmapheresis and should be offered for treating Guillain-Barré syndrome (GBS) in adults (Level A). IVIg is effective and should be offered in the long-term treatment of chronic inflammatory demyelinating polyneuropathy (Level A). IVIg is probably effective and should be considered for treating moderate to severe myasthenia gravis and multifocal motor neuropathy (Level B). IVIg is possibly effective and may be considered for treating nonresponsive dermatomyositis in adults and Lambert-Eaton myasthenic syndrome (Level C). Evidence is insufficient to support or refute use of / / ig in the treatment of immunoglobulin M paraprotein-associated neuropathy, inclusion body myositis, polymyositis, diabetic radiculoplexoneuropathy, or Miller Fisher syndrome, or in the routine treatment of postpolio syndrome or in children with GBS (Level U). IVIg combined with plasmapheresis should not be considered for treating GBS (Level B). More data are needed regarding IVIg efficacy as compared with other treatments/treatment combinations. Most studies concluded IVIg-related serious adverse effects were rare. Given the variable nature of these diseases, individualized treatments depending on patient need and physician judgment are important. Neurology ${ }^{\circledR}$ 2012;78:1009-1015

\section{GLOSSARY}

AAN = American Academy of Neurology; $\mathbf{A E}=$ adverse effect; $\mathbf{C I}=$ confidence interval; $\mathbf{C I D P}=$ chronic inflammatory demyelinating polyneuropathy; FDA $\Rightarrow$ Food and Drug Administration; GBS = Guillain-Barré syndrome; IBM = inclusion body myositis; IgM = immunoglobulin M; INCAT = Inflammatory Neuropathy Cause and Treatment Score; IVIg = IV immunoglobulin; LEMS = Lambert-Eaton myasthenic syndrome; $\mathbf{M G}=$ myasthenia gravis; $\mathbf{M M N}=$ multifocal motor neuropathy; $\mathbf{M P}=$ methylprednisolone; MRC = Medical Research Council; NDS = neurologic disability scale; QMG = Quantitative Myasthenia Gravis score; $\mathbf{R C T}=$ randomized controlled trial.

IV immunoglobulin (IVIg) is used to treat a range of immune-mediated neurologic diseases. The US Food and Drug Administration (FDA) approved IVIg for use in Guillain-Barré syndrome (GBS) and chronic inflammatory demyelinating polyneuropathy (CIDP), but IVIg use for non-FDA-approved indications is common. Although IVIg appears to be well tolerated in many patients, hypercoagulability and renal failure are of concern.

This American Academy of Neurology (AAN) evidence-based guideline summarizes the evidence and makes recommendations regarding IVIg use in treating patients with neuromuscular disorders.

\section{DESCRIPTION OF THE ANALYTIC PROCESS}

The Therapeutics and Technology Assessment subcommittee (appendix e-1 on the Neurology ${ }^{\circledR}$ Web site at www.neurology.org) selected panelists on the basis of expertise in IVIg use or familiarity with the guideline process, or both. A literature search of MEDLINE, Web of Science, and EMBASE databases from 1966 to 2009 was conducted, using the

From the Yale University/VA Connecticut Healthcare System (H.S.P.), New Haven, CT; Johns Hopkins Medical Institute (V.C.), Baltimore, MD University of Toronto (H.K.), Toronto, Canada; Cleveland Clinic (A.D.R.-G.), Cleveland, OH; and Stanford University (Y.T.S.), Palo Alto, CA. Approved by the Therapeutics and Technology Assessment Subcommittee on January 3, 2011; by the Practice Committee on February 7, 2011; and by the AAN Board of Directors on December 12, 2011.

Disclosure: Author disclosures are provided at the end of the article. 
search term "immunoglobulin" and one of the following: myasthenia gravis, GBS, neuropathy, CIDP, multifocal motor neuropathy, polymyositis, dermatomyositis, diabetic neuropathy, diabetic radiculoplexoneuropathy, postpolio syndrome, paraproteinemic neuropathy, Lambert-Eaton myasthenic syndrome, Miller Fisher syndrome, inclusion body myositis.

At least 2 panelists reviewed each abstract result; articles were included if they were therapeutic studies relevant to the efficacy, safety, tolerability, or IVIg mode of use in humans. Reviews and meta-analyses were reviewed to ensure inclusion of all relevant published studies. Studies were rated according to the AAN therapeutic classification of evidence scheme (appendix e-2); recommendations were linked to the strength of the evidence (appendix e-3). Disagreements on article classification were resolved by discussion. All data analyzed are presented in tables e-1 to e- 14 .

ANALYSIS OF EVIDENCE Guillain-Barré syndrome. Is IVIg effective in GBS in children? Data comparing IVIg with placebo in GBS treatment are limited. This may be due to the well-accepted use of plasmapheresis as treatment. ${ }^{1}$ One Class II study examined 21 children with mild GBS able to walk 5 meters unassisted. ${ }^{2}$ Fourteen children received treatment of 1 $\mathrm{g} / \mathrm{kg}$ over 2 days; the remaining patients received no treatment (no blinding or sham treatment was used). There was no difference between groups in the primary outcome measure of disability at the disease nadir. Some secondary measures favored the IVIg group (time to improvement $p<0.001$, disability duration $p<$ 0.05 , disability at 4 weeks $p<0.25$ ).

In another study (Class III), ${ }^{3} 18$ children were assigned to either IVIg $1 \mathrm{~g} / \mathrm{kg} / \mathrm{day}$ for 2 days or no treatment. Although the primary outcome was not well-defined, the interval from onset to maximum weakness (IVIg mean 9.3 days vs placebo mean 12.5 days, $p<0.05$ ), the interval from maximum weakness to improvement (IVIg 7.5 days vs placebo 11.8 days, $p<0.05$ ), and hospitalization length (IVIg mean 16.5 days vs placebo 23.8 days, $p<0.05$ ) all favored IVIg.

Due to differing outcome measures and small sample size in Class III studies, the benefit of IVIg in children with GBS is uncertain.

Clinical context. There is a lack of randomized, controlled studies of IVIg in children with GBS to show efficacy; however, most experts consider it a reasonable treatment option in children on the basis of its effectiveness in adults with GBS.

Is IVIg as effective as plasmapheresis in GBS in adults? Two Class I studies compared IVIg with plasmapheresis in GBS in adults. ${ }^{4,5}$ The first Class I study ${ }^{4}$ enrolled 150 patients with GBS and random- ized patients to 5-day treatment with either 5 volumes of plasma exchange or $0.4 \mathrm{~g} / \mathrm{kg} /$ day of IVIg. Patients were randomized within 14 days of symptom onset. Primary outcome measure was a 1-grade improvement on a 7 -point disability scale at 4 weeks (functional scale based on ability to walk and breathe independently, with $0=$ normal and $6=$ death). Fifty-three percent of patients given IVIg achieved a 1 -grade improvement at week 4 vs $34 \%$ given plasmapheresis $(p=0.024)$. Additionally, plasmapheresis patients took 41 days to reach 1 grade of improvement, and IVIg patients took 27 days $(~ p=$ 0.05 ). Although the examiner was unblinded, the outcome criteria were judged to be objective.

A Class I study ${ }^{5}$ enrolled 379 patients in a randomized, single-blind trial comparing IVIg, plasmapheresis, and plasmapheresis followed by IVIg within 14 days of GBS onset. The study found no significant difference among the 3 groups. The study was powered to demonstrate a 0.5 -point difference in disability grade with a $95 \%$ confidence level.

Three Class III studies did not change the above recommendations. ${ }^{6-8}$

All trials comparing IVIg with plasmapheresis used a standard of 5 exchanges, with $250 \mathrm{~mL} / \mathrm{kg}$ total volume exchanged in the control arm. Older studies varied widely in the number of exchanges (2 to 8) used. In our view, these comparison trials involved a satisfactory plasmapheresis regimen to test IVIg effectiveness and satisfy the AAN criteria for Class I noninferiority or equivalence trials (appendix e-2).

Is steroid an effective adjunctive treatment in patients with GBS treated with IVIg? One Class I study compared IVIg with IVIg plus methylprednisolone (MP). ${ }^{9}$ A total of 233 patients were randomized to receive IVIg $0.4 \mathrm{~g} / \mathrm{kg} /$ day for 5 days plus either MP (500 mg/day for adults or $8 \mathrm{mg} / \mathrm{kg} /$ day for children) or placebo. Primary outcome measure was disability improvement by 1 grade or more at 4 weeks. No significant difference was found $56 \%$ of patients in IVIg and 68\% in IVIg/MP groups, odds ratio 1.68, $95 \%$ confidence interval $[\mathrm{CI}] 0.97$ to $2.88, p=$ $0.06)$. This study was not adequately powered to exclude a meaningful benefit (risk difference $12.1 \%$, CI $-0.6 \%$ to $24.3 \%$ ).

What is the optimal IVIg dosing for GBS? One Class I study examined the role of 2 IVIg regimens for treating GBS. ${ }^{10}$ In this study, 39 patients were randomized to receive IVIg $0.4 \mathrm{~g} / \mathrm{kg} /$ day for 3 days or 6 days. Primary outcome was time to walk 5 meters. There was no significant difference in primary outcome (84 days vs 131 days in favor of 6-day treatment); however, the study lacked the statistical precision to exclude a clinically important difference. Subgroup analysis of patients who were ventilator dependent 
showed a better outcome with the longer IVIg regimen (86 days vs 152 days in favor of 6-day, $p=$ $0.04)$.

\section{Conclusions.}

1. Based on conflicting primary outcome measures, IVIg benefit is uncertain in children with GBS.

2. Based on 2 Class I studies, IVIg is as efficacious as plasmapheresis for treating GBS in adults. Because plasmapheresis is established as effective GBS treatment, ${ }^{1}$ we conclude that IVIg also has established effectiveness.

3. Based on one adequately powered Class I study, the combination of plasmapheresis and IVIg is probably not better than either treatment alone.

4. Based on one underpowered Class I study, evidence is insufficient to support or exclude a benefit of adding MP to IVIg in GBS.

5. Data are insufficient to make a recommendation on optimal IVIg dosing.

Recommendations. There is insufficient evidence to support or refute the effectiveness of IVIg in children with GBS (Level U). IVIg should be offered to treat GBS in adults (Level A). IVIg combined with plasmapheresis should not be considered for treating GBS (Level B). Evidence is insufficient to recommend MP in combination with IVIg (Level U).

Clinical context. Many experts consider it reasonable treatment to use IVIg for GBS in childrengiven its effectiveness in the same disease in adults.

Chronic inflammatory demyelinating polyneuropathy. Two Class I and 2 Class II studies compared IVIg with placebo in treating CIDP. ${ }^{114}$ In a Class I crossover study, ${ }^{11} 117$ patients received a loading dose of $2 \mathrm{~g} / \mathrm{kg}$ and then $1 \mathrm{~g} / \mathrm{kg}$ of IVIg or placebo (albumin) every 3 weeks. Patients were switched to the alternate treatment for worsening or no improvement. Patients completing 24 weeks of the first period were rerandomized to a single dose of IVIg or placebo. Primary endpoint was the percentage of patients who improved 1 or more points on the Inflammatory Neuropathy Cause and Treatment Score (INCAT). More patients improved in the IVIg group $(54 \%$ IVIg vs $21 \%$ placebo, $p=0.0002$ ). Four patients dropped out of the study. Adverse effects (AEs) occurred at a higher rate in the IVIg group $(55 \%$ vs $17 \%)$, but the number of serious AEs was low and similar in the 2 groups.

Another Class I study ${ }^{12}$ randomized 53 untreated patients with CIDP to IVIg or placebo. IVIg $1 \mathrm{~g} / \mathrm{kg}$ was administered on days 1,2 , and 21 . Primary outcome was change from baseline of the average muscle score (based on a modified Medical Research Council [MRC] scale expanded to 10 points) at day 42 .
This increased by 0.63 in the IVIg group and decreased by -0.1 in the placebo group ( $p=0.006$ ).

Two Class II studies ${ }^{13,14}$ comparing IVIg with placebo demonstrated IVIg benefits comparable with those shown in the Class I studies.

One Class II double-blind study compared oral prednisolone with IVIg for CIDP. ${ }^{15}$ Thirty-two patients were randomized to receive either IVIg followed by prednisolone or prednisolone followed by IVIg. Patients were treated with prednisolone $60 \mathrm{mg}$ tapered to $10 \mathrm{mg}$ over 6 weeks or IVIg $2 \mathrm{~g} / \mathrm{kg}$ given over 1 or 2 days. Analysis of 24/32 patients completing both treatment phases showed that both groups improved when compared with baseline, although there was no significant difference between the 2 treatment groups. The study, however, was not designed and powered to demonstrate equivalence of the 2 treatments.

\section{Conclusions.}

1. Based on 2 Class I studies, IVIg is effective for the long-term treatment of CIDP.

2. Data are insufficient to address the comparative efficacy of prednisolone and IVIg in treating CIDP.

Recommendation. IVIg should be offered for the long-term treatment of CIDP (Level A).

Clinical context. Dosing, frequency, and duration of IVIg for CIDP may vary depending on the clinical assessment. Data are insufficient to address the comparative efficacy of other CIDP treatments (e.g., steroids, plasmapheresis, immunosuppressants). Experts have identified that there may be overuse of IVIg in longterm care of CIDP. We were unable to evaluate this question using available randomized trial data.

Myasthenia gravis. One Class I study ${ }^{16}$ examined 24 patients with myasthenia gravis (MG) with worsening weakness treated with IVIg $2 \mathrm{~g} / \mathrm{kg}$ vs 27 patients on placebo. The Quantitative Myasthenia Gravis score (QMG) was better at 14 days in the IVIg group $(-2.54$ vs -0.89 in placebo, $p=0.047)$. In a subgroup analysis stratified for severity, IVIg was effective in patients with moderate or severe MG (QMG difference of -3.39 over placebo, $95 \%$ CI -5.88 to $-0.90, p=0.01)$ but produced no discernable difference in patients with mild MG.

One Class III study ${ }^{17}$ showed no difference in primary measures between IVIg and placebo, but assessment time (42 days) was unlikely to show benefit.

Two comparative Class III studies randomized patients with MG to treatment with either IVIg or plasmapheresis. ${ }^{18,19}$ One study ${ }^{18}$ compared the 2 treatments in 12 patients, using a single-blind, crossover design, and found no difference between the 2 groups, but the study is likely underpowered. A 
larger study randomized 46 patients to IVIg and 41 to plasmapheresis ${ }^{19}$ and was powered to detect a $50 \%$ difference in myasthenic muscular score (scale $0-100$ [normal]). The primary outcome assessment was unblinded. There was no difference in treatment efficacy at 15 days.

\section{Conclusions.}

1. Based on one Class I study, IVIg is probably effective in treating patients with MG.

2. Evidence is insufficient to compare the efficacy of IVIg and plasmapheresis in treating MG.

Recommendation. IVIg should be considered in the treatment of MG (Level B).

Clinical context. This recommendation was based on studies involving primarily moderately or severely affected patients. The benefits and risks of this medication should be weighed carefully in patients with mild MG. Further studies of IVIg efficacy in MG are warranted due to the few randomized trials and small study size to date.

Multifocal motor neuropathy. In one Class II randomized controlled trial (RCT), ${ }^{20} 19$ patients with multifocal motor neuropathy (MMN) were randomized to receive either IVIg $0.5 \mathrm{~g} / \mathrm{kg} / \mathrm{day}$ for 5 days or placebo monthly for 3 consecutive months. Patients who did not respond in one treatment arm were switched to the alternate treatment for an additional 3 months, with final evaluation after the treatment period. Primary endpoint was improvement in MRC sumscore at month 4 . Seven of 9 patients who received IVIg responded to treatment as compared with 2 of 9 who received placebo $(p=0.03)$. Most AEs were mild and occurred in the IVIg group. Subgroup analysis indicated a higher response rate in patients who had been treated with IVIg in the past vs those who had never received IVIg before the study (8 of 9 patients with prior IVIg treatment responded vs 5 of 9 without; there was one dropout from the previously untreated group).

A Class II crossover study ${ }^{21}$ enrolled 16 patients to receive either IVIg $0.4 \mathrm{~g} / \mathrm{kg}$ for 5 days or placebo. Patients were assessed at 28 days using a modified neurologic disability scale (NDS; summed score of 26 muscles, each rated $0-4)$ as the primary outcome measure, with grip strength, change in conduction block, and subjective assessment as secondary measures. All patients completed the study; 13/16 had mild AEs. NDS scores improved $6.7 \pm 3.3$ in the patients treated with IVIg and worsened $2.1 \pm 3.0$ in the placebo patients $(p=0.038)$. All secondary outcome measures improved in the IVIg group. Eleven of 16 patients reported improvement on IVIg; none reported improvement on placebo. A small Class II study ${ }^{22}$ treated 5 patients with IVIg $0.4 \mathrm{~g} / \mathrm{kg} /$ day or placebo for 5 days in a crossover design. Primary outcome measure was quantitative muscle strength in 2 muscles. Strength improved 28 days after treatment $(p<0.05)$.

Conclusion. Based on consistent results from 3 Class II studies, IVIg is probably effective for MMN treatment.

Recommendation. IVIg should be considered for the treatment of MMN (Level B).

Clinical context. $\mathrm{MMN}$ is a chronic disease requiring ongoing treatment. No data are available to address optimal treatment dosing, interval, and duration.

Neuropathy associated with IgM paraprotein. In a Class I study, 22 patients with paraproteinemic neuropathy associated with immunoglobulin M ( $\operatorname{IgM})$ were randomized to receive either IVIg $2 \mathrm{~g} / \mathrm{kg}$ or placebo in a double-blind crossover trial. ${ }^{23}$ There was no significant difference in the primary endpoint, the INCAT disability scale, at 2 weeks. A Class II study evaluated 11 patients with IgM paraprotein. ${ }^{24}$ All were treated with IVIg $2 \mathrm{~g} / \mathrm{kg}$ or placebo monthly for 3 consecutive months. No significant difference was detected in the MRC sumscore and other outcome measures over the treatment period.

Conclusion. Based on 1 Class I study and 1 Class II study, IVIg is possibly ineffective for the treatment of IgM paraprotein-associated neuropathy. A modest benefit cannot be excluded due to each study's small sample size.

Recommendation. Evidence is insufficient to assess the role of IVIg in treating neuropathy associated with IgM paraprotein (Level U).

Dermatomyositis. One Class II crossover study ${ }^{25}$ evaluated 15 adults with biopsy-confirmed dermatomyositis who did not respond to prior treatments. Five outcome measures were used, none designated as primary. Summed MRC scores of 18 muscles (maximum normal score 90) improved by 8.0 after 3 months of treatment $(p<0.018)$; the neuromuscular symptom score (maximum normal score 60$)$ improved by $7.3(p<$ 0.035). Repeat muscle biopsies showed improved cytoarchitecture and reduced muscle inflammation. Activities of daily living scores based on Barthel Index improved to 100 from a low of 65; rash as assessed by photography improved "markedly."

Conclusion. Based on 1 Class II study, IVIg is possibly effective for the treatment of nonresponsive dermatomyositis in adults.

Recommendation. IVIg may be considered for the treatment of nonresponsive dermatomyositis in adults (Level C).

Inclusion body myositis. In one Class I study ${ }^{26} 19$ patients with inclusion body myositis (IBM) were randomized and completed treatment with either IVIg or placebo. The primary outcome measure was 
a summed modified MRC score of 20 muscles $(0-10$ for each muscle; maximum normal score $=200$ ). The score improved by a mean of 4.2 after 3 months of IVIg, whereas that of the placebo group declined by 2.7. The difference was nonsignificant. A secondary outcome measure, quantitative strength testing, did not show a significant effect. Another secondary outcome measure, duration of swallowing, showed significant difference favoring IVIg in some cases $(p<0.05)$, but no correction was made for multiple comparisons.

A Class I crossover study ${ }^{27}$ assessed the addition of high-dose prednisone to IVIg. All 37 patients were pretreated with a tapering regimen of oral prednisone and then randomized to receive either IVIg with continuing prednisone or placebo with prednisone. The primary outcome measures were the summed modified MRC score of 13 muscles and quantitative strength testing 2 and 3 months after beginning infusion treatments. There was no difference between treatment and placebo groups in either measure. Regarding secondary measures, the number of necrotic muscle fibers declined ( $p<0.01$ ), and the number of endomysial inflammation foci decreased $(p<$ $0.005)$ in the IVIg/prednisone group when compared with controls.

In a Class II study ${ }^{28}$ of 22 patients, each patient received either IVIg or placebo for 6 months, followed by crossover to the other arm. Multiple outcome measures were used without a designated primary outcome. There was no difference in the summed modified MRC score, but improvement was seen in the neuromuscular symptom score (IVIg 6.2 vs placebo 1.2 during the first 6 months, IVIg 2.6 vs placebo -0.6 during the second 6 months, $p<$ $0.05)$. Other outcome measures were not significantly different between treatment groups.

Conclusion. Two Class I studies and 1 Class II study failed to demonstrate a consistent or significant clinical benefit of IVIg in treating IBM.

Recommendation. Evidence is insufficient to support or refute the use of IVIg in treating IBM (Level U).

Clinical context. There is presently no effective treatment for IBM.

Postpolio syndrome. Two Class I studies evaluated IVIg efficacy in patients with postpolio syndrome. ${ }^{29,30}$ The first study, a double-blind RCT, evaluated 142 patients with postpolio syndrome, most with severe weakness (Class IV or V based on the National Rehabilitation Postpolio Limb Classification). ${ }^{29}$ The 2 primary outcome measures were improved strength of a muscle believed to be significantly affected by postpolio (25\%-75\% of normal for age and sex) and improved quality of life as measured by the Short Form-36. The study found a significant improvement of $8.6 \%$ in the strength of the most affected muscle, but this did not reach a predetermined target of $15 \%$ for clinical significance. There was no significant effect on quality of life measures.

Another Class I, double-blind, randomized pilot study of 20 patients with postpolio syndrome was performed. ${ }^{30}$ There were 3 concurrent primary outcome measures: muscle strength as measured by fixed dynamometer on selected muscles, Fatigue Severity Scale, and pain as measured by visual analog scale and pain drawing instrument change at 3 months. The study showed that patients receiving IVIg had significantly improved pain levels at 3 months as compared with baseline; however, there were no effects on muscle strength or fatigue.

Conclusion. One Class I study showed a significant difference, but the difference was not clinically important for IVIg use on the most affected muscle in postpolio syndrome. One underpowered Class I study showed an effect of IVIg for pain in postpolio syndrome but no effect on strength or fatigue.

Recommendation. Evidence is insufficient to support or refute IVIg use in the routine treatment of postpolio syndrome (Level U).

Clinical context. There is presently no effective treatment for postpolio syndrome.

Other neuromuscular disorders. One Class II placebocontrolled crossover study evaluated IVIg efficacy in 10 patients with Lambert-Eaton myasthenic syndrome (LEMS). ${ }^{31}$ As compared with placebo, IVIg showed a modest benefit on vital capacity, limb strength, drinking time, and antibody titer. There are no controlled studies evaluating the effects of IVIg on polymyositis, diabetic polyradiculoplexoneuropathy, or Miller Fisher syndrome.

Conclusion. Based on 1 Class II study, IVIg is possibly effective in LEMS.

Recommendation. IVIg may be considered in the treatment of LEMS (Level C).

Adverse effects of IVIg. Eighteen of the 22 prospective studies reviewed recorded the number of serious and minor AEs from 632 patients receiving IVIg (total dose $2.0-2.5 \mathrm{~g} / \mathrm{kg}$ ). There were no IVIg-related deaths in these studies. Most studies concluded that IVIg was well-tolerated and AEs were either transient or manageable. Serious AEs related to IVIg were rare and included aseptic meningitis $(\mathrm{n}=3)$, urticaria $(\mathrm{n}=2)$, heart failure $(\mathrm{n}=1)$, myocardial infarction $(\mathrm{n}=1)$, and renal failure $(\mathrm{n}=1)$. These findings do not exclude the possibility of rare AEs such as stroke and thrombotic events, which have been previously reported with IVIg. It is important to screen for vas- 
cular risk factors before infusion and to monitor carefully during and after infusion. ${ }^{32-34}$ The most common IVIg-related AEs included headache (16.1\%), fever (6.6\%), mild hypertension (4.6\%), chills $(3.3 \%)$, nausea $(3.2 \%)$, asthenia $(1.4 \%)$, arthralgia $(1.3 \%)$, anorexia $(1.1 \%)$, dizziness $(1.1 \%)$, malaise (1.1\%), and transient hyperglycemia (1.1\%).

Clinical context. It is important to assess individual patient risk for AEs when considering IVIg therapy.

\section{RECOMMENDATIONS FOR FUTURE RESEARCH}

1. For most of the diseases examined here, alternative treatment modalities are available. Comparative studies may be helpful.

2. IVIg benefit is generally short lived; further, longterm studies might be useful.

3. Studies are needed to explore possible synergistic effects of adjunctive treatments such as immunosuppressants or plasmapheresis.

4. Few data are available on the optimal IVIg infusion frequency and cumulative dose.

5. A larger study of IVIg in patients with mild MG may be useful.

6. The issue of overtreatment of CIDP with TVIg, which may be a clinically significant issue, could be assessed with further observational studies.

\section{AUTHOR CONTRIBUTIONS}

Dr. Patwa: drafting/revising the manuscript, analysis or interpretation of data, contribution of vital reagents/tools/patients, acquisition of data. Dr. Chaudhry: drafting/revising the manuscript, study concept or design. Dr. Katzberg: drafting/revising the manuscript, analysis or interpretation of data, acquisition of data. Dr. Rae-Grant: drafting/revising the manuscript, analysis or interpretation of data. Dr. So: drafting/revising the manuscript, study concept or design, analysis or interpretation of data, acquisition of data, strat

\section{DISCLOSURE}

Dr. Patwa was an investigator in the ICE trial comparing IVIg with placebo for CIDP. Dr. Chaudhry serves on the editorial board of Neurologist; is an inventor on patent(s) re: Total Neuropathy Score (TNS) — a score for evaluating peripheral neuropathies, for which he receives technology royalties from Abbott, Johnson \& Johnson, and sanofi-aventis; receives publishing royalties for Harrison's Principles of Internal Medicine, 17th ed. (McGraw Hill Companies, Inc., 2008); estimates that $40 \%$ of his clinical effort is spent on nerve conduction studies; has given expert testimony for the Department of Health and Human Services Vaccine Injury Compensation program; and receives research support from the Neuropathy Association, Nutricia, and Insmed Inc. Dr. Katzberg has received funding for travel from the Muscular Dystrophy Association. Dr. Rae-Grant has received speaker honoraria from Biogen Idec, Teva Pharmaceutical Industries Ltd., and EMD Serono, Inc.; receives publishing royalties for Handbook of Multiple Sclerosis (Springer Healthcare, 2010); and has served on the speakers' bureau for Biogen Idec. Dr. So receives publishing royalties for Occupational \& Environmental Medicine (Appleton \& Lange, 2007), Occupational \& Environmental Medicine (Appleton \& Lange, 2007), and contributions to UpToDate; receives research support from the NIH (NIEHS, NINDS); and holds stock in Sartoris, Inc.

\section{DISCLAIMER}

This statement is provided as an educational service of the American Academy of Neurology. It is based on an assessment of current scientific and clinical information. It is not intended to include all possible proper methods of care for a particular neurologic problem or all legitimate criteria for choosing to use a specific procedure. Neither is it intended to exclude any reasonable alternative methodologies. The AAN recognizes that specific patient care decisions are the prerogative of the patient and the physician caring for the patient, based on all of the circumstances involved. The clinical context section is made available in order to place the evidence-based guidelines into perspective with current practice habits and challenges. No formal practice recommendations should be inferred.

\section{CONFLICT OF INTEREST}

The American Academy of Neurology is committed to producing independent, critical and truthful clinical practice guidelines (CPGs). Significant efforts are made to minimize the potential for conflicts of interest to influence the recommendations of this CPG. To the extent possible, the AAN keeps separate those who have a financial stake in the success or failure of the products appraised in the CPGs and the developers of the guidelines. Conflict of interest forms were obtained from all authors and reviewed by an oversight committee prior to project initiation. AAN limits the participation of authors with substantial conflicts of interest. The AAN forbids commercial participation in, or funding of, guideline projects. Drafts of the guideline have been reviewed by at least three AAN committees, a network of neurologists, Neurology ${ }^{\circledR}$ peer reviewers and representatives from related fields. The AAN Guideline Author Conflict of Interest Policy can be viewed at www.aan.com.

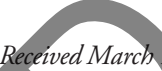

2011. Accepted in final form November 28, 2011.

\section{REFERENCES}

Cortese I, Chaudhry V, So YT, Cantor F, Cornblath DR, Rae-Grant A. Evidence-based guideline update: plasmapheresis in neurological disorders: report of the Therapeutics and Technology Assessment Subcommittee of the American Academy of Neurology. Neurology 2011;76: 294-300.

2. Korinthenberg R, Schess J, Kirschner J, Monting JS. Intravenously administered immunoglobulin in the treatment of childhood Guillain-Barré syndrome: a randomized trial. Pediatrics 2005;116:8-14.

3. Gurses N, Uysal S, Cetinkaya F, Ilek I, Kalayci AG. Intravenous immunoglobulin treatment in children with Guillain-Barré syndrome. Scand J Infect Dis 1995;27: 241-243.

4. van der Meche FG, Schmitz PI; for Dutch Guillain-Barré Study Group. A randomized trial comparing intravenous immune globulin and plasma exchange in Guillain-Barré syndrome. N Engl J Med 1992;326:1123-1129.

5. Hughes RAC, Swan AV; for Plasma Exchange/Sandoglobulin Guillain-Barré Syndrome Trial Group. Randomised trial of plasma exchange, intravenous immunoglobulin, and combined treatments in Guillain-Barré syndrome. Lancet 1997; 349:225-230.

6. Bril V, Ilse WK, Pearce R, et al. Pilot trial of immunoglobulin versus plasma exchange in patients with GuillainBarré syndrome. Neurology 1996;46:100-103.

7. Nomura T, Hamaguchi K, Hattori T, et al. A randomized controlled trial comparing intravenous immunoglobulin and plasmapheresis in Guillain-Barré syndrome. Neurol Therapeutics 2000;18:69-81.

8. Diener H-C, Haupt WF, Kloss TM, et al. A preliminary, randomized, multicenter study comparing intravenous immunoglobulin, plasma exchange, and immune adsorption in Guillain-Barré Syndrome. Eur Neurol 2001;46:107109.

9. van Koningsveld R, Schmitz PI, Meche FG, Visser LH, Meulstee J, van Doorn PA. Effect of methylprednisolone when added to standard treatment with intravenous im- 
munoglobulin for Guillain-Barré syndrome: randomised trial. Lancet 2004;363:192-196.

10. Raphael JC, Chevret S, Harboun M, Jars-Guincestre MC; for French Guillain-Barré Syndrome Cooperative Group. Intravenous immune globulins in patients with Guillain-Barré syndrome and contraindications to plasma exchange: 3 days versus 6 days. J Neurol Neurosurg Psychiatry 2001;71:235-238.

11. Hughes RA, Donofrio P, Bril V, et al. Intravenous immune globulin ( $10 \%$ caprylate-chromatography purified) for the treatment of chronic inflammatory demyelinating polyradiculoneuropathy (ICE study): a randomised placebo-controlled trial. Lancet Neurol 2008;7:136-144.

12. Mendell JR, Barohn RJ, Freimer ML, et al. Randomized controlled trial of IVIg in untreated chronic inflammatory demyelinating polyradiculoneuropathy. Neurology 2001; 56:445-449.

13. Hahn AF, Bolton CF, Zochodne D, Feasby TE. Intravenous immunoglobulin treatment in chronic inflammatory demyelinating polyneuropathy: a double-blind, placebocontrolled, cross-over study. Brain 1996;119:1067-1077.

14. Vermeulen M, van Doorn PA, Brand A, et al. Intravenous immunoglobulin treatment in patients with chronic inflammatory demyelinating polyneuropathy: a double blind, placebo controlled study. J Neurol Neurosurg Psychiatry 1993;56:36-39.

15. Hughes R, Bensa S, Willison H, et al. Randomized controlled trial of intravenous immunoglobulin versus oral prednisolone in chronic inflammatory demyelinating polyradiculoneuropathy. Ann Neurol 2001;50:195-201.

16. Zinman L, Ng E, Bril V. IV immunoglobulin in patients with myasthenia gravis: a randomized controlled trial Neurology 2007;68:837-841.

17. Wolfe GI, Barohn RJ, Foster BM, et al. Randomized, controlled trial of intravenous immunoglobulin in myasthenia gravis. Muscle Nerve 2002;26:549-552.

18. Ronager J, Ravnborg M, Hermansen I, Vorstrup S. Immunoglobulin treatment versus plasma exchange in patients with chronic moderate to severe myasthenia gravis. Artif Organs 2001:25:967-973.

19. Gajdos P, Chevret S, Clair B, Tranchant C, Chastang C. Clinical trial of plasma exchange and high-dose intravenous immunoglobulin in myasthenia gravis: Myasthenia Gravis Clinical Study Group. Ann Neurol 1997;41:789-796.

20. Leger JM, Chassande B, Musset L, Meininger V, Bouche $P$, Baumann N. Intravenous immunoglobulin therapy in multifocal motor neuropathy: a double-blind, placebocontrolled study. Brain 2001;124:145-153.

21. Federico P, Zochodne DW, Hahn AF, Brown WF, Feasby TE. Multifocal motor neuropathy improved by IVIg: randomized, double-blind, placebo-controlled study. Neurology 2000;55:1256-1262.

22. Azulay JP, Blin O, Pouget J, et al. Intravenous immunoglobulin treatment in patients with motor neuron syndromes associated with anti-GM1 antibodies: a double-blind, placebo-controlled study. Neurology 1994;44:429-432.
23. Comi G, Roveri L, Swan A, et al. A randomised controlled trial of intravenous immunoglobulin in IgM paraprotein associated demyelinating neuropathy. J Neurol 2002;249:13701377.

24. Dalakas MC, Quarles RH, Farrer RG, et al. A controlled study of intravenous immunoglobulin in demyelinating neuropathy with IgM gammopathy. Ann Neurol 1996;40:792795.

25. Dalakas MC, Illa I, Dambrosia JM, et al. A controlled trial of high-dose intravenous immune globulin infusions as treatment for dermatomyositis. N Engl J Med 1993;329: 1993-2000.

26. Dalakas MC, Sonies B, Dambrosia J, Sekul E, Cupler E, Sivakumar K. Treatment of inclusion-body myositis with IVIg: a double-blind, placebo-controlled study. Neurology 1997;48:712-716.

27. Dalakas MC, Koffman B, Fujii M, Spector S, Sivakumar K, Cupler E. A controlled study of intravenous immunoglobulin combined with prednisone in the treatment of IBM. Neurology 2001;56:323-327.

28. Walter MC, Lochmuller H, Toepfer M, et al. High-dose immunoglobulin therapy in sporadic inclusion body myositis: a double-blind, placebo-controlled study. J Neurol 2000;247:22-28.

29. Gonzalez H, Sunnerhagen KS, Sjoberg I, et al. Intravenous immunoglobulin for post-polio syndrome: a randomised controlled trial. Lancet Neurol 2006;5:493-500.

30. Farbu E, Rekand T, Vik-Mo E, et al. Post-polio patients treated with intravenous immunoglobulin: A doubleblinded randomized controlled pilot study. Eur J Neurol 2007;14:60-65.

31. Bain PG, Motomura M, Newsome-Davis J, et al. Effects of intravenous immunoglobulin on muscle weakness and calcium-channel autoantibodies in the Lambert-Eaton myasthenic syndrome. Neurology 1996;47:678-683.

32. Steinberger B, Coleman TA. Multiple complications of IVIg therapy in a patient with Guillain-Barré syndrome. Am J Hematol 2001;67:59.

33. Go RS, Call TG. Deep venous thrombosis of the arm after intravenous immunoglobulin infusion: case report and literature review of intravenous immunoglobulin-related thrombotic complications. Mayo Clin Proc 2000;75:83-85.

34. Caress JB, Hobson-Webb L, Passmore LV, Finkbiner AP, Cartwright MS. Case-control study of thromboembolic events associated with IV immunoglobulin. J Neurol 2009;256:339.

35. Van Den Berg LH, Kerkhoff H, Oey PL, et al. Treatment of multifocal motor neuropathy with high dose intravenous immunoglobulins: a double blind, placebo controlled study. J Neurol Neurosurg Psychiatry 1995;59:248-252.

36. Mariette X, Chastang C, Louboutin J-P, et al. A randomized clinical trial comparing interferon-alpha and intravenous immunoglobulin in polyneuropathy associated with monoclonal IgM. J Neurol Neurosurg Psychiatry 1997;63:28-34.

\section{Endorsed by the American Association of Neuromuscular and Electrodiagnostic Medicine on February 20, 2012.}




\title{
Neurology
}

\author{
Evidence-based guideline: Intravenous immunoglobulin in the treatment of \\ neuromuscular disorders [RETIRED]: Report of the Therapeutics and Technology \\ Assessment Subcommittee of the American Academy of Neurology \\ H.S. Patwa, V. Chaudhry, H. Katzberg, et al. \\ Neurology 2012;78;1009-1015 \\ DOI 10.1212/WNL.0b013e31824de293
}

This information is current as of March 26, 2012

\section{Updated Information \& Services}

Supplementary Material

\section{Citations}

Permissions \& Licensing

Reprints including high resolution figures, can be found at: http://n.neurology.org/content/78/13/1009.full

Supplementary material can be found at: http://n.neurology.org/content/supp1/2012/03/25/78.13.1009.DC1 http://n.neurology.org/content/supp1/2012/03/25/78.13.1009.DC2 http://n.neurology.org/content/suppl/2012/07/24/78.13.1009.DC3 http://n.neurology.org/content/suppl/2012/12/03/78.13.1009.DC4 http://n.neurology.org/content/supp1/2013/01/03/78.13.1009.DC5

This article has been cited by 5 HighWire-hosted articles: http://n.neurology.org/content/78/13/1009.full\#\#otherarticles

Information about reproducing this article in parts (figures,tables) or in its entirety can be found online at:

http://www.neurology.org/about/about_the_journal\#permissions

Information about ordering reprints can be found online:

http://n.neurology.org/subscribers/advertise

Neurology ${ }^{\circledR}$ is the official journal of the American Academy of Neurology. Published continuously since 1951, it is now a weekly with 48 issues per year. Copyright Copyright (? 2012 by AAN Enterprises, Inc.. All rights reserved. Print ISSN: 0028-3878. Online ISSN: 1526-632X.

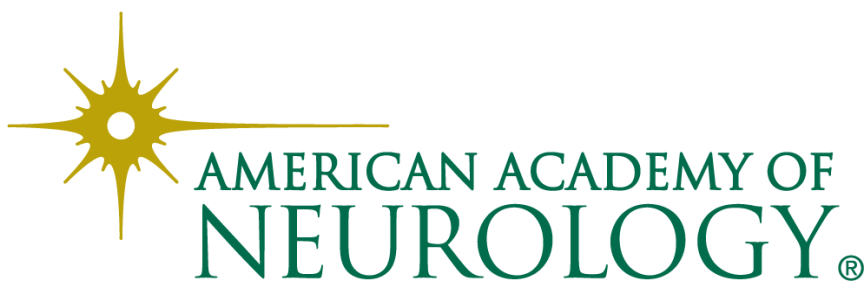

